Краткие сообщения

03

\title{
Численное изучение поступательной неравновесности в смеси Не и Хе методом Монте-Карло нестационарного статистического моделирования
}

\author{
(C) С.В. Куликов \\ Институт проблем химической фризики РАН, \\ 142432 Черноголовка, Московская область, Россия \\ e-mail: kuls@icp.ac.ru
}

(Поступило в Редакцию 13 января 2016 г. В окончательной редакции 14 ноября 2016 г.)

Изучены распределения пар частиц по относительным скоростям во фронте ударной волны для случая малой добавки Хе в Не. Оказалось, что величины распределений по относительным скоростям для пар атомов Хе значительно (вплоть до $10^{9}$ ) превышают их равновесные значения за волной в узкой зоне фронта при больших скоростях в области относительно малых (равных 2) чисел Маха ударной волны. Величины распределений пар из атомов Не и Хе по относительным скоростям таким свойством не обладают.

DOI: 10.21883/JTF.2017.06.44520.1735

\section{Введение}

Изучение поступательной неравновесности, а именно распределений пар частиц по относительным скоростям $(g)$ во фронте ударной волны очень важно для понимания пороговых физико-химических процессов инициируемых ударной волной. В [1] сделана попытка обобщения имеющихся в этой области результатов, полученных методом Монте-Карло нестационарного статистического моделирования (ММКНСМ) (или direct simulation Monte Carlo (DSMC) в англоязычной литературе). В [2] качественно были подтверждены результаты [1]. Причем представленные в [2] результаты были получены путем численного решения уравнения Больцмана.

Можно рассматривать в качестве меры неравновесности максимальные (обнаруженные при моделировании в различных точках фронта) для данного числа Маха ударной волны $(M)$ величины превышений парных распределений частиц по $g$ соответствующего равновесного распределения по $g$ за волной (MGD). Необходимо подчеркнуть, что величина превышения определяется как отношение этих значений распределений. Суммируя ранее полученные результаты, следует отметить, что при достаточно малом содержании примеси для распределений по $g$, в случае пар, состоящих из одной частицы легкого газа и одной частицы тяжелой примеси $\left(G_{L H}\right)$, величины MGD меньше, чем для случая пар, состоящих только из частиц тяжелой примеси $\left(G_{H H}\right)$. Кроме того, при уменьшении концентрации тяжелой примеси значения MGD стремятся к предельным значениям. Последнее очевидно в силу того, что в предельном случае тяжелые частицы перестают сталкиваться между собой.
Ниже представлены результаты моделирования для двух случаев малой добавки Хе в Не при различных значениях $M$. Приведенные ниже результаты несколько неожиданны, так как свидетельствуют о существовании сильной поступательной неравновесности при относительно малых числах М ударной волны.

\section{Методика моделирования}

Расчеты были проведены ММКНСМ [6]. Кратко процедура моделирования состоит в следующем. В первый момент времени модельные частицы, представляющие газовую смесь, имеют в соответствии с начальными условиями заданные скорости и распределены по ячейкам размера $\Delta x$, на которые разбито исследуемое пространство координат. Полагается, что столкновения парные и с определенной вероятностью происходят только между частицами, находящимися в одной ячейке.

Процесс эволюции рассматриваемой системы за интервал времени $\Delta t$ расщепляется на два этапа: 1) только перемещение частиц с неизменными скоростями в промежутке времени $\Delta t$; 2) только изменение скоростей частиц в результате их столкновений в этом же промежутке времени при их фиксированных положениях.

Данный метод моделирования позволяет получать решение уравнения Больцмана, избегая очень трудоемкого решения последнего и предоставляя наиболее полную и достоверную информацию. Кроме того, метод не имеет принципиальных ограничений по ширине спектра энергий столкновений, однако с ростом энергий рассматриваемых реакций резко возрастает вычислительная сложность задачи. Лишь относительно недавно развитие вычислительной техники предоставило возможность существенно продвинуться в область больших энергий. 
Рассматривалось одномерное пространство координат и трехмерное пространство скоростей. Иными словами, отслеживалось только движение частиц вдоль потока. А при моделировании столкновений частиц рассматривались все три составляющие их скоростей. Рассматривалась смесь Не и тяжелого компонента Хе. Атомы рассматривались как абсолютно упругие твердые сферы. Среднее число частиц в ячейке перед фронтом $\mathrm{N}=12000$. Полагалось, что значения параметров потока на границах отрезка моделирования перед и за ударной волной равновесные. Был реализован мелкозернистый параллелизм [6] на восьми процессорах ЭВМ МВС100К Межведомственного суперкомпьютерного центра.

Отношение масс атомов Не и Хе, как широко принято, полагалось 1:33, а диаметров 1:2.25 [7]. Последнее отношение получено из измерения взаимной диффузии рассматриваемых газов. Необходимо отметить, что в [8] были экспериментально измерены профили концентраций в смеси $97 \% \mathrm{He}+3 \% \mathrm{Xe}$ при $M=3.89$. Приведенное выше отношение диаметров атомов Не и Хе не позволяет воспроизвести эти профили не только в наших, но и в расчетах ряда других исследователей. При этом следует отметить, что полученные в [4,9-11] профили хорошо согласуются между собой, несмотря на то что расчеты были выполнены различными методами. Но в эти расчеты были заложены модели абсолютно жестких и упругих сфер. И все эти упомянутые расчеты дают гораздо меньшую сепарацию компонентов во фронте. От результатов расчетов, приведенных выше, отличаются результаты представленные в [2], которые были получены на основе численного решения уравнения Больцмана с использованием потенциала Леннард-Джонса. Приведенные в [2] профили концентраций в смеси $97 \% \mathrm{He}+3 \%$ Хе при $M=3.89$ хорошо совпадают с измеренными в [8]. Это настораживает, так как в $[4,11]$ представлено сравнение профилей концентраций в смеси $97.3 \% \mathrm{He}+2.7 \% \mathrm{Ar}$ (аналогично малая примесь тяжелого газа в легком) при $M=2.07$, полученных в результате численного моделирования, с экспериментальными [12]. Причем из данных $[4,11]$ следует, что лучшее соответствие с экспериментом было получено для модели абсолютно жестких и упругих сфер, а модель точечных центров отталкивания давала более пологий профиль $\mathrm{Ar}$ (большую сепарацию компонентов во фронте). В [2] сравнение с экспериментом для смеси $\mathrm{He}-\mathrm{Ar}$ было выполнено только для смесей с содержанием $\mathrm{Ar} 11.5 \%$ и выше для $M \approx 1.6$ по-прежнему с использованием потенциала Леннард-Джонса. А для таких смесей можно использовать как модель абсолютно жестких и упругих сфер, так и модель точечных центров отталкивания или полагать одинаковые параметры взаимодействия атомов как для Не, так и для $\mathrm{Ar}$ и получать неплохое соответствие с экспериментом [4,11]. В [2] не было проведено сравнение с результатами опытов [12]. Так что вопрос остается открытым. К сожалению, никто из других экспериментаторов не померил профили компонентов в условиях эксперимента [8] или близких к этим условиям.

\section{Результаты моделирования}

В таблице приведены величины MGD для $G_{L H}$ и $G_{H H}$. Результаты представлены для различных $M$ и для объемных концентраций Хе 1 и $0.5 \%$.

Имеет место сильное увеличение (на 4 порядка) максимального MGD для $G_{H H}$ при уменьшении концентрации Хе с 1 до 0.5\%. При этом максимум MGD наблюдается при более низком $M(M=2)$. Следует отметить, что если в [8] приведены правильные профили концентраций, то расчеты с диаметрами атомов, приводящими к этим профилям, дадут еще большие значения MGD [1].

Ниже представлены в качестве иллюстрации результаты, полученные для $M=2$ при концентрации Хе $0.5 \%$. На рис. 1 показаны профили относительных концентраций $n_{i}^{0}=\left(n_{i}-n_{i 1}\right) /\left(n_{i 2}-n_{i 1}\right)$ и относительных кинетических температур $T_{i}^{0}=\left(T_{i}-T_{1}\right) /\left(T_{2}-T_{1}\right)$. Здесь индексы 1 и 2 относятся к параметрам газа перед и за ударной волной. Расстояние $x$ нормировано на среднюю длину свободного пробега в смеси перед волной $\lambda$, во всех расчетах $\Delta x=0.15 \lambda$. Следует отметить, что профили $n_{i}^{0}$ и $T_{i}^{0}$, полученные при концентрации Хе $1 \%$, практически совпадают с профилями при $0.5 \%$.

Значения MGD

\begin{tabular}{c|c|c|c|c|c|c|c}
\hline \multicolumn{2}{c|}{$M$} & 1.8 & 2 & 2.5 & 3 & 5 & 7 \\
\hline \multirow{2}{*}{$1 \%$} & $G_{L H}$ & - & 200 & 100 & 10 & 20 & 10 \\
\cline { 2 - 8 } & $G_{H H}$ & - & 40 & 6000 & 2000 & $2 \cdot 10^{5}$ & $4 \cdot 10^{4}$ \\
\hline \multirow{2}{*}{$0.5 \%$} & $G_{L H}$ & 10 & 6 & 20 & - & - & - \\
\cline { 2 - 8 } & $G_{H H}$ & 100 & $10^{9}$ & $8 \cdot 10^{6}$ & - & - & -
\end{tabular}

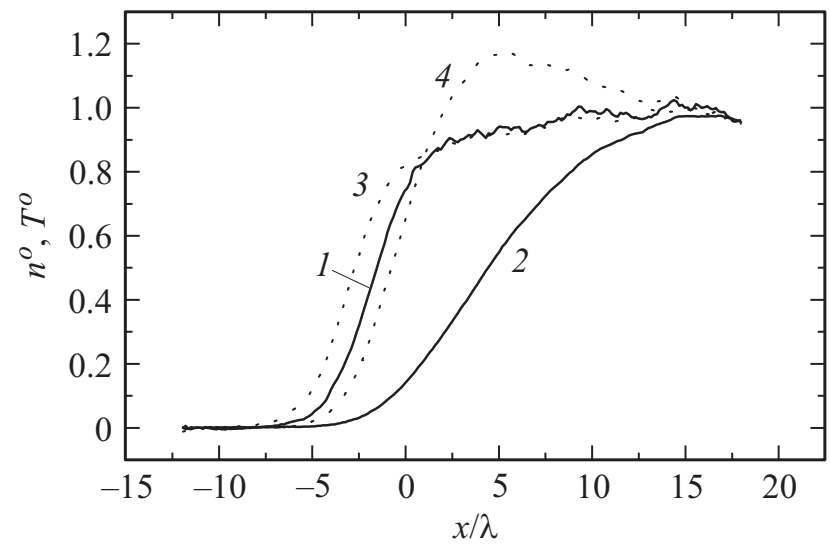

Рис. 1. Относительные профили концентраций и температур компонентов: $1-n_{\mathrm{He}}^{0}, 2-n_{\mathrm{Xe}}^{0}, 3-T_{\mathrm{He}}^{0}, 4-T_{\mathrm{Xe}}^{0}$. 


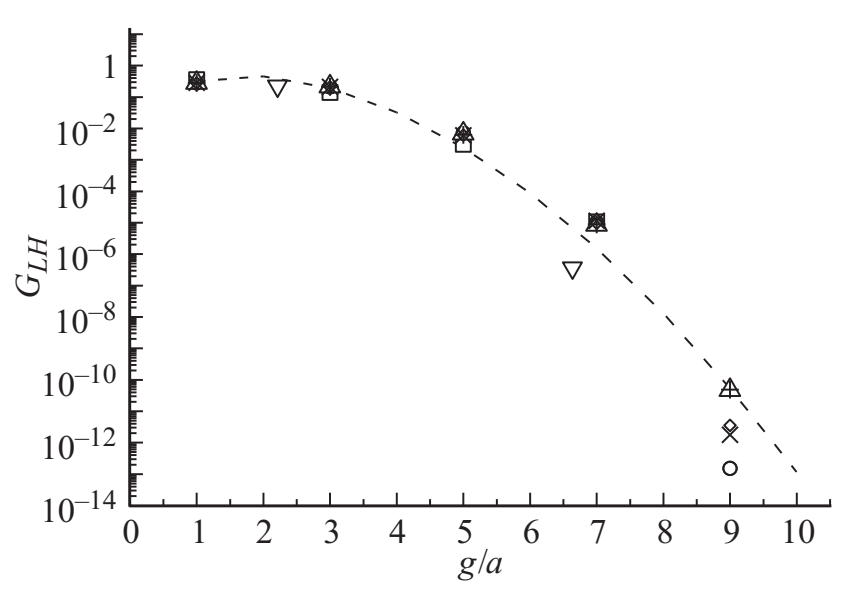

Рис. 2. Распределения $G_{L H}$ по $g$ во фронте ударной волны (пояснение в тексте).

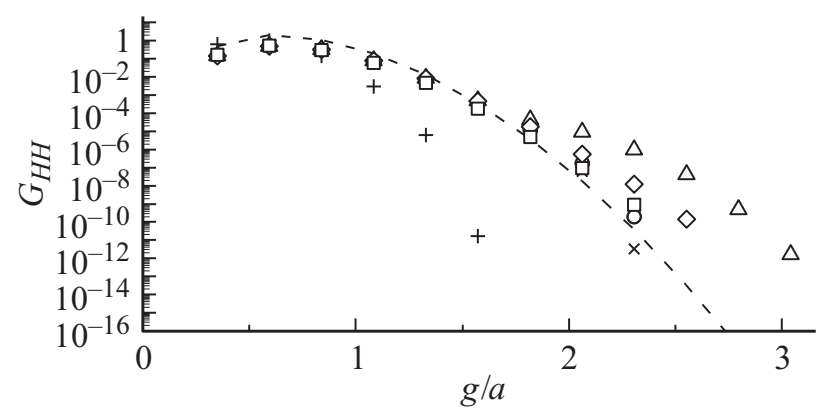

Рис. 3. Распределения $G_{H H}$ по $g$ во фронте ударной волны (пояснение в тексте).

Кроме того, на рис. 2 представлены $G_{L H}$ : --- равновесное за фронтом, $\nabla-$ при $x=-11.42, \square-$ при $x=-3.08, \circ-$ при $x=-0.08, \Delta-$ при $x=1.42, \diamond-$ при $x=2.92, \times-$ при $x=4.42,+$ - при $x=17.32$. И на рис. 3 представлены $G_{H H}$ : -.- - равновесное за фронтом: + - при $x=-11.42, \times-$ при $x=1.42$, опри $x=-0.2 .92, \Delta-$ при $x=4.42, \diamond-$ при $x=5.92$, $\square-$ при $x=17.32$. Величины $g$ нормированы на $a$, где $a-$ скорость звука в потоке перед фронтом. Эти распределения нормированы так, что интегралы от них по $g / a$ равны единице. Представление о точности значений данных распределений можно получить из [1]. При высоких $g$ на хвостах распределений это примерно 50\%.

Как видно, во время пересечения газом фронта распределения $G_{L H}$ не сильно превышают даже в области высоких $g$ равновесные значения $G_{L H}$ за волной. Напротив, величины $G_{H H}$ значительно (вплоть до $10^{9}$ ) превышают их равновесные значения за волной в узкой зоне фронта при больших $g$. Но моделирование показало, как и следовало ожидать, очень малую вероятность молекулярных столкновений тяжелого компонента с тяжелым. Следовательно, высокоэнергетические хвосты $G_{H H}$ образуются во фронте главным образом в результате столкновений атомов тяжелого компонента Хе с атомами Не.

\section{Заключение}

Оказалось, что величины $G_{H H}$ значительно (вплоть до $10^{9}$ ) превышают их равновесные значения за волной в узкой зоне фронта при больших $g$. Это довольно неожиданный результат. При реализации таких столкновений с высокой энергией относительного движения не исключено сильное возбуждение внутренних степеней свободы Не или Хе и даже их ионизация.

Автор благодарен О.Н. Терновой за помощь в обработке результатов.

Работа выполнена при поддержке РФФИ (грант 12-0801266a).

\section{Список литературы}

[1] Куликов С.В. // Матем. модел. 1999. Т. 11. № 3. С. 96-104.

[2] Додулат О.И., Клосса Ю.Ю., Черемисин Ф.Г. // Физикохимическая кинетика в газовой динамике. Электронный журнал. 2013. Т. 4. Вып. 1. http://www.chemphys.edu.ru).

[3] Генич А.П., Куликов С.В., Манелис Г.Б. и др. // Журн. вычисл. матем. и матем. физ. 1986. Т. 26. № 12. С. 1839.

[4] Genich A.P., Kulikov S.V., Manelis G.B., Chereshnev S.L. // Sov. Tech. Rev. B. Therm. Phys. 1992. Vol. 4. N 1. P. 1. Генич А.П., Куликов С.В., Манелис Г.Б., Черешнев С.Л. Поступательная релаксация в ударных волнах в газах. Препринт ИХФЧ АН СССР. Черноголовка. 1991.

[5] Емельянов А.В., Еремин А.В., Куликов С.В. // ЖТФ. 2013. Т. 83. Вып. 5. С. 24-29.

[6] Куликов С.В., Берзигияров П.К. // Вычисл. методы и програм. 2002. Т. 3. № 2. С. 51-56.

[7] Bird G.A. Molecular gas dynamics. Oxford: Clarendon Press, 1976. Берд Г. Молекулярная газовая динамика. М.: Мир. 1981.

[8] Gmurczyk A.S., Tarczynski M., Walenta Z.A. Proceedings of the 11h Rarefied Gasdynemics Symp, Cannes, France. July 1978. 3. 333. Cd. R. Campargue, 1979.

[9] Schmidt B., Worner M. // Acta Mech. 1983. Vol. 46. N 1-4. P. 49-55.

[10] Руев Г.А., Фомин В.М., Шавалиев М.Ш. / Численные и аналитические методы в динамике разреженных газов. Tp. VIII Всес. конф. по динамике разр. газов. Москва, 1985. M.: 1986. C. 12-17.

[11] Куликов С.В., Соловьева М.Е. // Журн. вычисл. матем. и матем. физ. 1988. Т. 28. № 12. С. 1867-1873.

[12] Center R.E. // Phys. Fluid. 1967. Vol. 10. N 8. P. 1777-1784. 